\title{
Leishmania mexicana metacaspase is a negative regulator of amastigote proliferation in mammalian cells
}

\author{
E Castanys-Muñoz ${ }^{1}$ E Brown ${ }^{1}$, GH Coombs ${ }^{2}$ and JC Mottram ${ }^{\star, 1}$
}

Metacaspases (MCAs) are caspase family cysteine peptidases that have been implicated in cell death processes in plants, fungi and protozoa. MCAs have also been suggested to be involved in cell cycle control, differentiation and clearance of aggregates; they are virulence factors. Dissecting the function of MCAs has been complicated by the presence in many organisms of multiple $M C A$ genes or limitations on genetic manipulation. We describe here the creation of a $M C A$ gene-deletion mutant $(\Delta m c a)$ in the protozoan parasite Leishmania mexicana, which has allowed us to dissect the role of the parasite's single MCA gene in cell growth and cell death. $\Delta$ mca parasites are viable as promastigotes, and differentiate normally to the amastigote form both in in vitro macrophages infection and in mice. $\Delta$ mca promastigotes respond to cell death inducers such as the drug miltefosine and $\mathrm{H}_{2} \mathrm{O}_{2}$ similarly to wild-type (WT) promastigotes, suggesting that MCAs do not have a caspase-like role in execution of L. mexicana cell death. $\Delta$ mca amastigotes replicated significantly faster than WT amastigotes in macrophages and in mice, but not as axenic culture in vitro. We propose that the Leishmania MCA acts as a negative regulator of amastigote proliferation, thereby acting to balance cell growth and cell death.

Cell Death and Disease (2012) 3, e385; doi:10.1038/cddis.2012.113; published online 6 September 2012

Subject Category: Immunity

Apoptosis is a process of programmed cell death (PCD) required in multicellular organisms for normal tissue development and homoeostasis, as it eliminates cells that are damaged, infected or no longer needed. ${ }^{1}$ A number of the biochemical and morphological events that define apoptosis have been described in the kinetoplastid parasites Trypanosoma brucei and Leishmania, which cause the important neglected tropical diseases African trypanosomiasis and leishmaniasis, respectively. ${ }^{2}$ Leishmania has a digenetic life cycle, with two main morphological forms alternating between two hosts. Motile flagellated promastigotes proliferate as freeliving cells in the sand fly. ${ }^{3}$ They are transmitted by the bite of the sand fly and, once inside a mammalian host, they are taken up by phagocytes, where they transform into intracellular amastigotes. They multiply and the disease progresses due to further infection of macrophages with amastigotes. ${ }^{4}$

The apoptosis-like processes reported to occur in these kinetoplastid parasites include cell shrinkage, DNA fragmentation, activation of peptidases, mitochondrial depolarisation, release of cytochrome $C$, phosphatidylserine (PS) exposure and translocation of endonuclease $\mathrm{G}^{2,5}$ Such morphological and biochemical features have been described for different Leishmania species under a variety of stress stimuli, including nitric oxide or reactive oxygen species (ROS) produced by the host, hydrogen peroxide, heat shock, and the leishmanicidal drugs miltefosine (MLF) and camptothecin (CPT).$^{6-12}$ Current thinking proposes that unicellular parasitic protozoa such as Leishmania undergo apoptotic-like PCD as an altruistic trait to control parasite numbers to prolong survival of the host and parasite. $^{13}$ Although evolutionary theory can provide a framework to understand why unicellular organisms might undergo $P C D$, questions remain about the molecular mechanisms responsible for induction of such cell death. In particular, caspases, which have an essential role in the execution of apoptosis in higher eukaryotes, are absent from genomes of Leishmania and Trypanosoma. ${ }^{14,15}$ Thus, it remains crucial to rigorously analyse possible cell death pathways and effector proteins regulating cell death in Leishmania and related protozoa to identify which cell death mechanisms are truly operating.

Metacaspases (MCAs) are cysteine peptidases grouped in clan CD, family 14 . They are present in plants, yeast and protozoan parasites, but absent from mammals, and are distantly related to caspases, thus have been promoted to have caspase-like biological functions. ${ }^{16}$ MCAs have a conserved caspase-like histidine-cysteine catalytic dyad, yet they have different substrate specificity from caspases and prefer arginine/lysine in the $\mathrm{P} 1$ position rather than the aspartic acid residue preferred by caspases. ${ }^{17-19}$ MCAs are activated by calcium and autocatalytic processing has been

\footnotetext{
${ }^{1}$ Wellcome Trust Centre for Molecular Parasitology, Institute of Infection, Immunity and Inflammation, College of Medical, Veterinary and Life Sciences, University of Glasgow, Glasgow G12 8TA, UK and ${ }^{2}$ Strathclyde Institute of Pharmacy and Biomedical Sciences, University of Strathclyde, Glasgow G4 ORE, UK

*Corresponding author: JC Mottram, Wellcome Trust Centre for Molecular Parasitology, University of Glasgow, 120 University Place, Glasgow G12 8 TA, UK. Tel: + 440141330 3745; Fax: + 440141330 8269; E-mail: jeremy.mottram@ glasgow.ac.uk

Keywords: metacaspase; caspase; cysteine peptidase; programmed cell death

Abbreviations: BLE, phleomycin-resistance gene; HYG, hygromycin-resistance gene; MCA, metacaspase; MLF, miltefosine; PCD, programmed cell death; PEMs, peritoneal exudate macrophages; TMRM, tetramethylrhodamine methyl ester perchlorate; TSN, Tudor staphylococcal nuclease; WT, wild type

Received 05.6.12; revised 06.7.12; accepted 06.7.12; Edited by A Stephanou
} 
described for many MCAs, although this processing is not apparently necessary for enzymatic activity. ${ }^{17,18,20}$ The structural basis for the substrate specificity and calcium activation has recently become apparent from the X-ray structure of the MCA2 from $T$. brucei. $^{21}$ Few natural substrates of MCAs are known, although the finding that Tudor staphylococcal nuclease (TSN), cleaved by the Picea abies MCA mcll-Pa during embryogenesis and induced PCD, is a substrate for both MCAs and caspases has been used as evidence of an evolutionary conserved cell death pathway in plants and animals. ${ }^{22}$

The report that the yeast MCA had a role in cell death ${ }^{23}$ triggered a series of similar studies investigating such a role for other MCAs. ${ }^{23}$ Subsequently, however, a number of other functions have been established for yeast MCA, from cell cycle involvement to clearance of insoluble aggregates. ${ }^{24,25}$ In the plant Arabidopsis thaliana, MCAs have antagonistic influences on cell death - with AtMC1 promoting cell death, whereas AtMC2 inhibits it. ${ }^{26}$ The single MCA of Leishmania major was described to have an important role in the cell cycle, ${ }^{27}$ while it has also been reported to be involved in oxidative stress-induced cell death. ${ }^{28}$ The MCA of Leishmania donovani has been proposed to have a role in cell death pathways $^{29,30}$ and also the cell cycle. ${ }^{30}$

The aim of this study was to provide additional insights into the role of MCA of Leishmania and related organisms by generating an MCA-deficient mutant. This achievement with Leishmania mexicana has allowed us to evaluate critically the possible participation of the single MCA in cell death and cell growth pathways. The data demonstrate that MCA is not essential for Leishmania survival or differentiation and, while not ruling out a role in regulated cell death, provide no evidence for such a role. In contrast, MCA clearly is implicated in the regulation of parasite replication inside the mammalian host.

\section{Results}

Generation of L. mexicana MCA null mutants. We were unsuccessful in our attempts in a previous study to generate L. major MCA gene deletion mutants and MCA overexpression lines had a severe growth defect, which we interpreted as being due to the importance of MCA levels to cellular replication. ${ }^{27}$ To be able to analyse the role of MCAs more fully, we decided to investigate the protein of $L$. mexicana. L. mexicana is an excellent experimental model as it can be cultivated in vitro in both promastigote and amastigote-like life cycle stages and its virulence can be assessed both in macrophage infection studies in vitro and the formation of murine lesions in vivo. Having identified the MCA gene of L. mexicana, which encodes a predicted protein of $48 \mathrm{kDa}$ that has $89 \%$ identity with $L$. major MCA, we used the Nucleofector, a newly developed system that provides highefficiency transfection while being protective of cellular function, to genetically manipulate MCAs in L. mexicana. To our surprise, this improved transfection procedure allowed us to generate $M C A$ null mutant cell lines ( $\Delta$ mca; Figure 1a). Southern blot analysis confirmed the replacement of both MCA alleles, the $0.8-\mathrm{kb}$ fragment detected in the wild type (WT) with a $5^{\prime} \mathrm{FR}$ probe in an Agel/Sacll-digested genomic DNA being replaced in $\Delta m c a$ parasites by $2.2-$ or 5-kb fragments corresponding to the hygromycin-resistance gene $(H Y G)$ or phleomycin-resistance gene $(B L E)$ casettes, respectively (Figure $1 \mathrm{~b}$ ). A probe comprising $500 \mathrm{bp}$ of the MCA open reading frame (ORF) was used to confirm the absence of the gene (Figure $1 \mathrm{~b}$, lower panel, lane 3 ). The absence of MCA was further confirmed by western blotting, using an affinity purified antibody raised against a peptide containing the C-terminal 15 amino acids of $L$. major MCA (designated $\alpha$ LmjMCA, 27; Figure 1c, lane 2, arrowed). MCA was reintroduced into the ribosomal locus, to give $\triangle m c a:: M C A$. Re-expression of MCA was confirmed by western blot analysis, where MCA was detected at slightly higher levels of expression in the re-expressor cell line than in the WT promastigotes (Figure 1c, lane 3). The antibody recognised a number of cross-reacting proteins in $\Delta m c a$, but also processed products (lane 3). L. mexicana promastigotes overexpressing either MCA (designated WT (MCA)) or an active site mutant in which the two cysteines in the active site have been mutated to glycines (designated WT $\left(\mathrm{MCA}^{\mathrm{C} 201-202 \mathrm{G}}\right)$ ) were also generated. Western blot analysis using the MCA antibody showed an increased level of the protein and the processed products in both WT (MCA) and WT $\left(\mathrm{MCA}^{\mathrm{C} 201-202 \mathrm{G}}\right)$ (Figure 1d, lanes 4 and 5) compared with WT, in which equivalent proteins cannot be visualised at this exposure (lane 1) and $\triangle m c a:$ MCA (lane 3).

Slight variations in growth of the different lines of promastigotes were observed in vitro, $\triangle$ mca::MCA reached a higher density at day 6 than WT whereas WT (MCA $^{\mathrm{C201-202G}}$ ) obtained a lower density than WT (Figure 1e). The DNA content of WT, $\triangle m c a$, WT (MCA) and $\triangle m c a:: M C A$ promastigotes was analysed by fluorescence-activated cell sorting (FACS); each had normal DNA content with two peaks corresponding to cells in G1 and G2/M (data not shown). In contrast to promastigotes, axenic amastigotes of $\Delta m c a$ grew to a higher density than WT at day 9, whereas amastigotes of WT (MCA) had a markedly reduced growth rate compared with WT. Re-expression of MCAs in the $\triangle m c a:$ : MCA largely restored the growth of axenic amastigotes to WT levels (Figure 1f). To assess the subcellular location of L. mexicana MCA, the expression of MCAs in the different cell lines was analysed by immunofluorescence using $\alpha$ LmjMCA. $^{27} \mathrm{MCA}$ was found to be mainly cytoplasmic, being present in punctate structures throughout the cell body in WT, WT (MCA) and $\triangle m c a:: M C A$; its absence from $\triangle m c a$ promastigotes served as a control (Figure 2).

MCA-deficient mutants are more virulent. The ability of the different lines to infect and multiply within peritoneal exudate macrophages (PEMs) was assessed. The percentage of infected PEMs with WT, $\Delta m c a$ and $\triangle m c a:: M C A$ did not significantly differ at 3,24 or $72 \mathrm{~h}$. A small increase was observed at $120 \mathrm{~h}$ for $\Delta \mathrm{mca}$, most likely due to lysis of some heavily infected macrophages and infection of additional PEMs by the released amastigotes (Figure 3a). The intracellular proliferation, however, was greater for $\Delta \mathrm{mca}$ than WT with there being significantly greater numbers of intracellular amastigotes at 72 and $120 \mathrm{~h}$ (Figure 3b). We also investigated whether overexpressing MCAs had any effect upon virulence in PEMs. The number of PEMs infected 
a

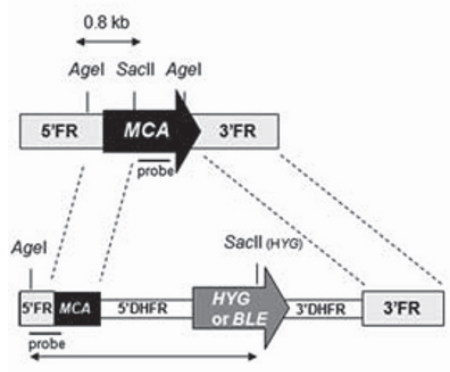

$22 \mathrm{~kb}$ (HYG) or $5 \mathrm{~kb}$ (BLE) b

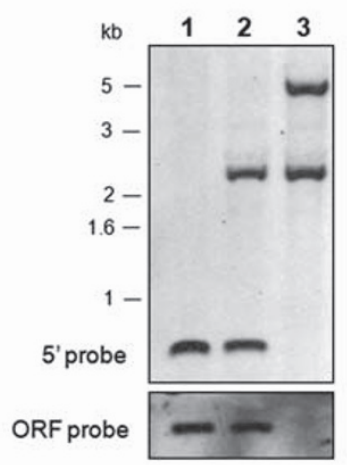

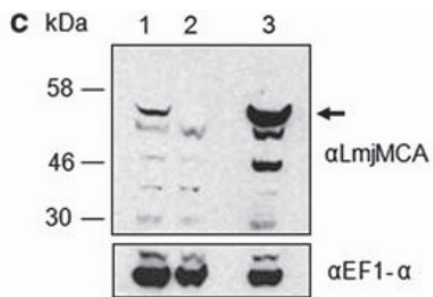

d

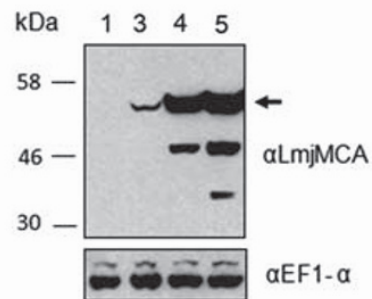

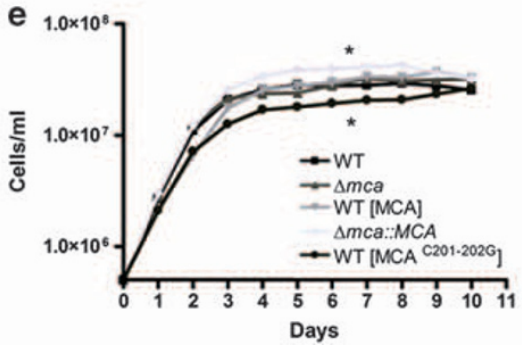

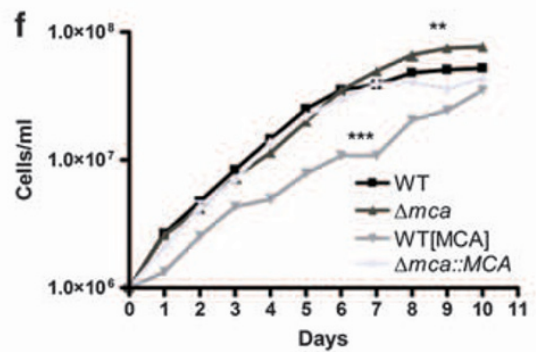

Figure 1 Generation of MCA null mutants. (a) Schematic representation of the L. mexicana WT MCA locus and the constructs used for targeted gene replacement. ORFs are shown as arrows, $5^{\prime}$ FR plus $5^{\prime}$ end of $M C A$, and $3^{\prime} F R$ DNA sequences used for gene targeting are shown as boxes. Restriction sites within the WT locus and the construct are shown, and the predicted sizes after DNA digestion are indicated for both native and modified MCA locus. DHFR, dihydrofolate reductase gene. (b) Southern blot analysis. Genomic DNA was digested with Agel/Sacll and hybridised with a labelled DNA probe comprising the $5^{\prime}$ region used for gene targeting (upper) or an ORF probe (lower). Molecular mass markers are shown on the left. WT (lane 1), heterozygote (lane 2) and $\Delta m c a$ (lane 3). (c and d) Western blot analysis. Whole-cell lysates of $L$. mexicana

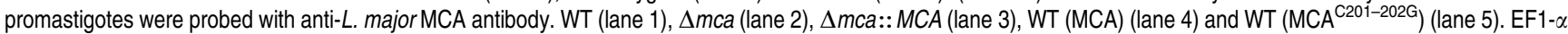

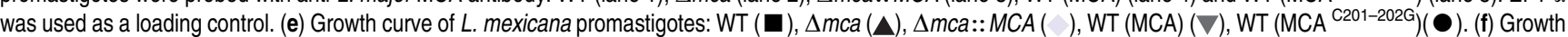
curve of $L$. mexicana axenic amastigotes: WT $(\mathbf{\square}), \Delta m c a(\boldsymbol{\Delta}), \Delta m c a:: M C A(>), W T(M C A)(\nabla) .{ }^{* \star *},{ }^{* \star}$ and ${ }^{*}$ significant differences compared with WT $\left(t\right.$-test, ${ }^{* \star \star} P<0.001$, ${ }^{\star \star} P<0.01$ and $\left.{ }^{*} P<0.05\right)$

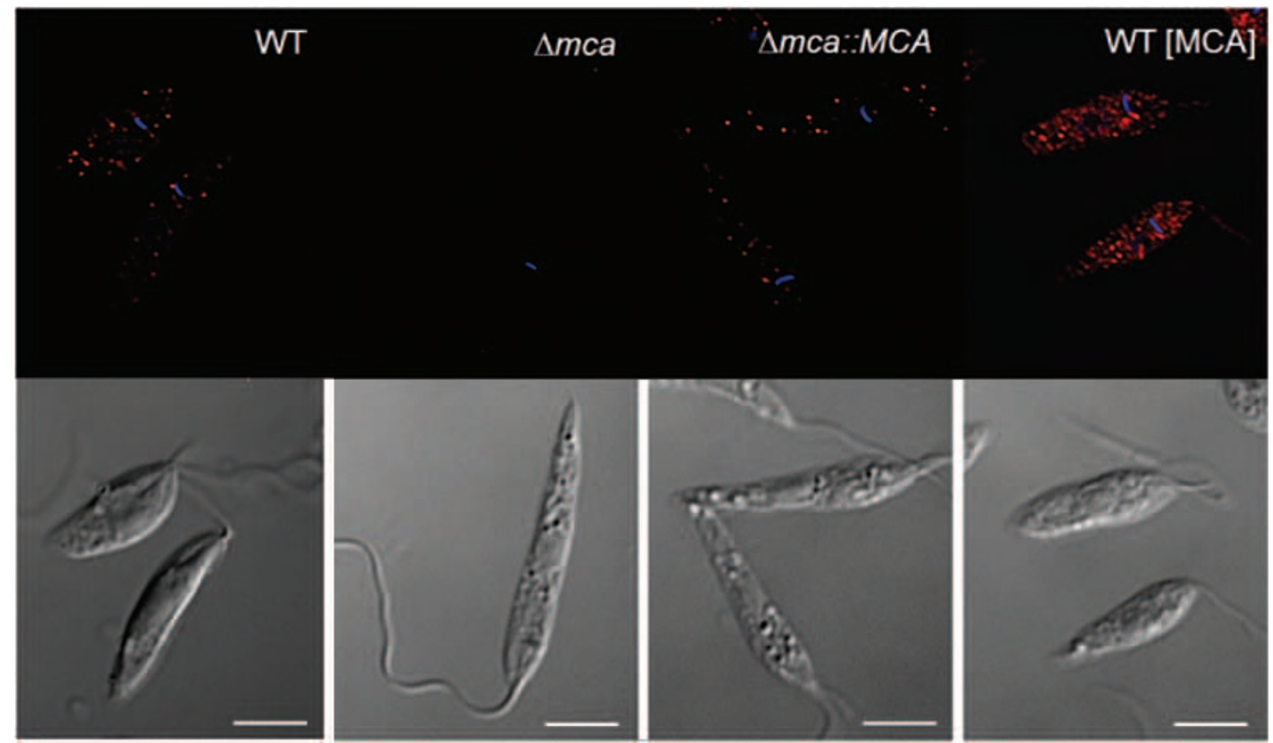

Figure 2 MCA localisation. Immunofluorescence analysis performed on L. mexicana promastigotes, using anti-LmjMCA antibody and Alexa fluor 594-conjugated anti-rabbit antibody (red). Upper panels, immunofluorescence on cell lines as indicated. Lower panels, differential interference contrast. DAPI staining of the nucleus and kinetoplast is shown in blue. Scale bar represents $5 \mu \mathrm{m}$ 

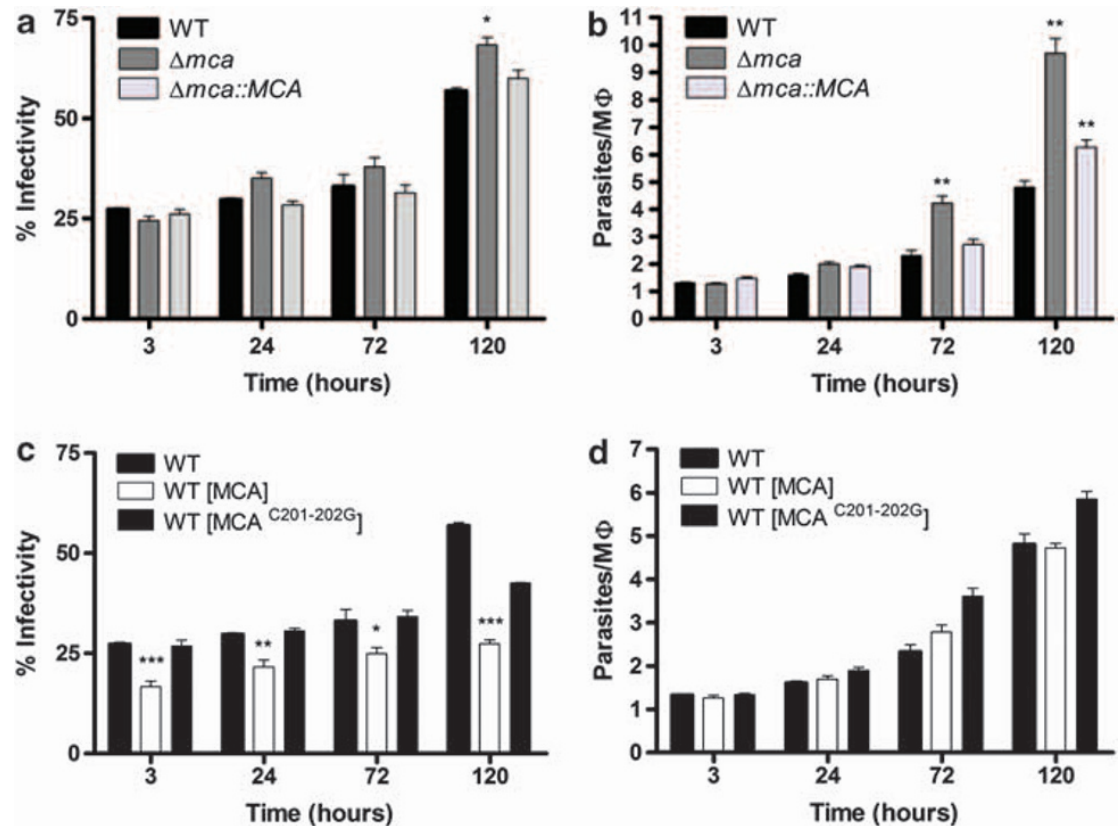

Figure 3 In vitro macrophage infectivity assay with promastigotes. PEMs were infected with $L$. mexicana stationary-phase promastigotes at a ratio of five promastigotes to one PEM for $3 \mathrm{~h}$ at $32^{\circ} \mathrm{C}$ and $5 \% \mathrm{CO}_{2}$. The parasite load was determined by counting the number of infected PEMs $(\mathbf{a}$ and $\mathbf{c})$ and the number of intracellular parasites ( $\mathbf{b}$ and $\left.\mathbf{d}\right)$ after $3,24,72$ and $120 \mathrm{~h}$ of incubation. Data show the percentage of infected macrophages and the number of parasites per macrophage (means $\pm \mathrm{s}$.d from four points) of a representative experiment out of three independent infections. Significant differences compared with WT are indicated as follows $\left(t\right.$-test, ${ }^{* * *} P<0.001 ;{ }^{* \star} P<0.01$; and $\left.{ }^{*} P<0.05\right)$

with WT (MCA) was significantly lower than with WT (Figure 3c), although intracellular multiplication was similar (Figure 3d). The infectivity of WT (MCA ${ }^{\mathrm{C} 201-202 G}$ ) was not significantly different from WT, indicating that the lower infectivity phenotype of WT (MCA) is dependent on the proteolytic activity of MCAs.

We extended our study using amastigotes isolated from animal lesions. The results are consistent with those using promastigotes, but the differences between the mutants and WT were more pronounced with greater differences being found at more time points (Figures $4 \mathrm{a}$ and $b$ ). In addition, WT (MCA) multiplied more slowly within PEMs than did WT (Figure 4b). To further corroborate the findings, we also used axenic amastigotes that had been differentiated from promastigotes in vitro, which yielded results very similar to those with lesion-derived amastigotes (Figures 4c and d).

The ability of the different lines to produce lesions in mice was also investigated. Stationary-phase promastigotes $\left(2 \times 10^{6}\right)$ were injected into the footpad of BALB/c mice and lesion development monitored. Footpad measurements indicated that $\Delta m c a$ formed significantly larger lesions than WT and $\triangle m c a:: M C A$ parasites over an 8-week period (Figure 5a), whereasWT (MCA) parasites formed significantly smaller lesions than WT or WT (MCA ${ }^{\mathrm{C} 201-202 G}$ ) (Figure 5b). These data show that $L$. mexicana lacking MCAs are more virulent than WT in macrophages in vitro and in mice in vivo and that overexpression of MCAs has the opposite effect of reducing parasite growth, the latter phenotype being dependent on the expression of an active MCA.
Analysis of MCA-deficient mutants. It has been reported that overexpression of Leishmania MCAs enhances sensitivity to inducers of cell death, ${ }^{28,29}$ including heat shock, ${ }^{8,12}$ hydrogen peroxide, ${ }^{6} \mathrm{ROS}^{7}$ and drugs such as MLF and CPT. ${ }^{9,10}$ Thus, we tested whether L. mexicana $\Delta$ mca would be protected against cell death induced by some of these agents. A method commonly used to assess cell death in Leishmania is propidium iodide (PI) staining, which has been applied to detect cells undergoing necrosis. In addition, annexin V (Ann V) labelling, which has been used extensively as a cell death marker in Leishmania, ${ }^{8,31,32}$ is presumed to bind PS. Promastigotes were cultured in the presence of $20 \mu \mathrm{M}$ MLF for $15 \mathrm{~h}$ whereupon PI staining and Ann V labelling was assessed. In all, $96 \%$ of WT and $\Delta m c a$ promastigotes were Ann $\mathrm{V}^{-} \mathrm{PI}^{-}$under normal growth conditions, whereas after treatment with MLF only $37 \%$ of WT promastigotes remained Ann $\mathrm{V}^{-} \mathrm{PI}^{-}$. In total, $72 \%$ of MLF-treated $\Delta m c a$ were Ann $\mathrm{V}^{-} \mathrm{PI}^{-}$, showing that $\Delta m c a$ were less susceptible to MLF-induced cell death (Figure 6a). Recently it has been reported that Leishmania promastigotes have very low levels of $P S,{ }^{33}$ so Ann $\mathrm{V}$ may not be a particularly suitable marker for regulated cell death in this parasite. However, the differences in Ann $V$ labelling detected between WT and $\Delta m c a$ parasites treated with MLF suggests that biochemical changes have occurred to the surface of the parasites and these are worthy of further investigation. $\Delta$ mca had a higher $\mathrm{IC}_{50}$ for MLF than WT, a difference reversed by re-expression of MCAs (Figure 6b). Importantly, however, $\Delta$ mca were not similarly less susceptible than WT to other stimuli reported to trigger cell death in 

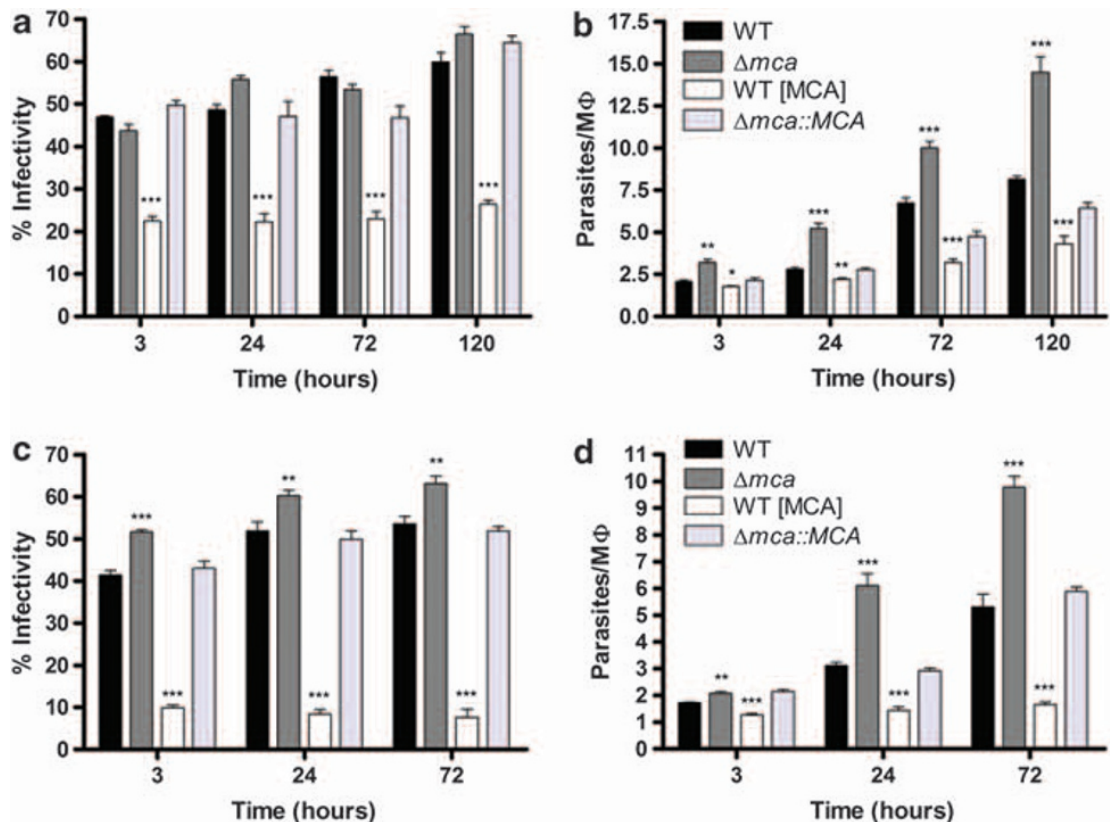

Figure 4 In vitro macrophage infectivity assay with amastigotes. PEMs were infected with L. mexicana amastigotes from lesions (a, b) or axenic amastigotes, (c, d) at 2: 1 or $1: 1$ ratio, respectively, for $3 \mathrm{~h}$. The percentage of infected PEMs (a and $\mathbf{c}$ ) and the number of amastigotes per PEMs ( $\mathbf{b}$ and $\mathbf{d}$ ) were determined after $3,24,72$ and $120 \mathrm{~h}$ of incubation. Data show the percentage of infected macrophages and the number of parasites per macrophage (means \pm s.d from four points) of a representative experiment out of three independent infections. Significant differences compared with WT are indicated as follows ( $t$-test, ${ }^{* * *} P<0.001 ;{ }^{* \star} P<0.01$; and $\left.{ }^{*} P<0.05\right)$
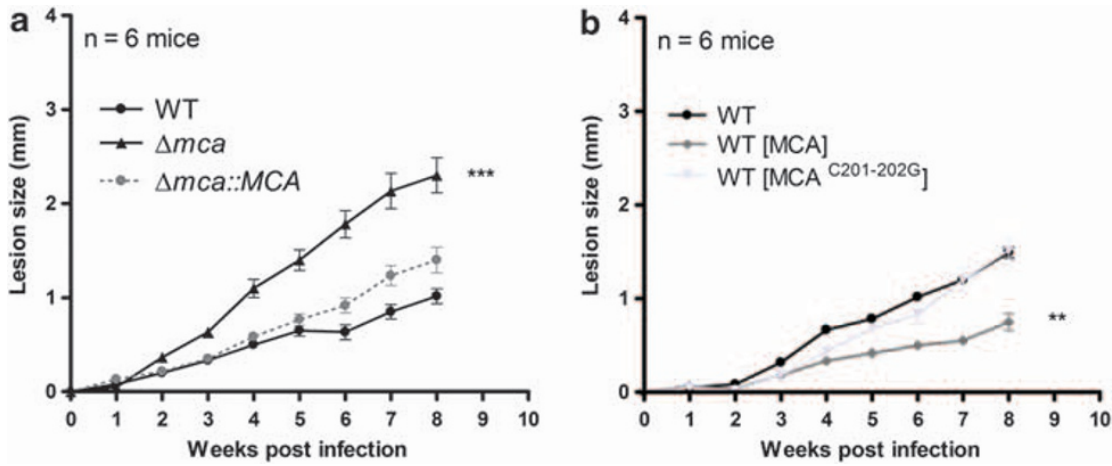

Figure 5 Infectivity to mice. (a and $\mathbf{b})$ Infection of BALB/c mice with stationary-phase $L$. mexicana promastigotes. Groups of mice $(n=6)$ were infected with $2 \times 10^{6}$ parasites in the right footpad. The ability of the different cell lines to produce lesions in mice was monitored using a calliper. Data shown represent the mean lesion size $\pm \mathrm{s}$.d from groups of six mice. Significant differences are indicated are indicated as follows (t-test, ${ }^{* \star \star} P<0.001 ;{ }^{* \star} P<0.01$ )

Leishmania, such as oxidative stress (data not shown) or CPT (Figure 6b).

To determine the ability of $\Delta m c a$ amastigotes to persist within macrophages in the presence of MLF, PEMs were infected with WT and $\Delta$ mca promastigotes for $24 \mathrm{~h}$ and then treated with $30 \mu \mathrm{M}$ MLF. The percentage of infected macrophages and the number of amastigotes per macrophage were determined after 3 days of drug treatment. The infectivity and number of amastigotes of $\Delta m c a$ per macrophage was significantly higher than WT (Supplementary Figures S1A and $\mathrm{B}$ ) under the same conditions. This suggests that $\Delta m c a$ parasites replicate better in macrophages, even in the presence of MLF. To investigate the susceptibility of $\Delta m c a$ amastigotes to killing by macrophages, PEMs were infected with WT and $\Delta m c a$ promastigotes for $3 \mathrm{~h}$ and subsequently stimulated with LPS and IFN- $\gamma$ (Supplementary Figures S1C and D). $\Delta$ mca were found to be significantly more resistant to killing by activated macrophages compared with WT.

It has been reported that MLF causes $L$. donovani promastigote cell death through mitochondrial dysfunction ${ }^{11}$ and that overexpression of MCAs in $L$. major resulted in impaired mitochondrial membrane potential, ${ }^{28}$ thus we reasoned that lack of MCAs may also affect the mitochondrion. Our results show that the mitochondrial membrane potential of $\Delta$ mca promastigotes was $36 \%$ that of WT, whereas overexpression of MCAs had a smaller effect with the mitochondrial membrane potential of WT (MCA) being $87 \%$ of WT (Figure $6 \mathrm{c}$ ). In spite of the reduced mitochondrial membrane potential of $\Delta m c a$ promastigotes, they were viable, grew at rates comparable to those of WT (Figure 1e) and had 


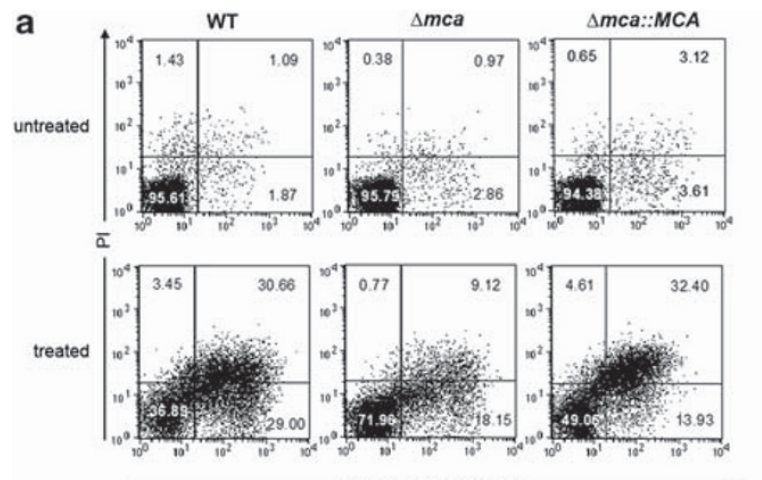

b
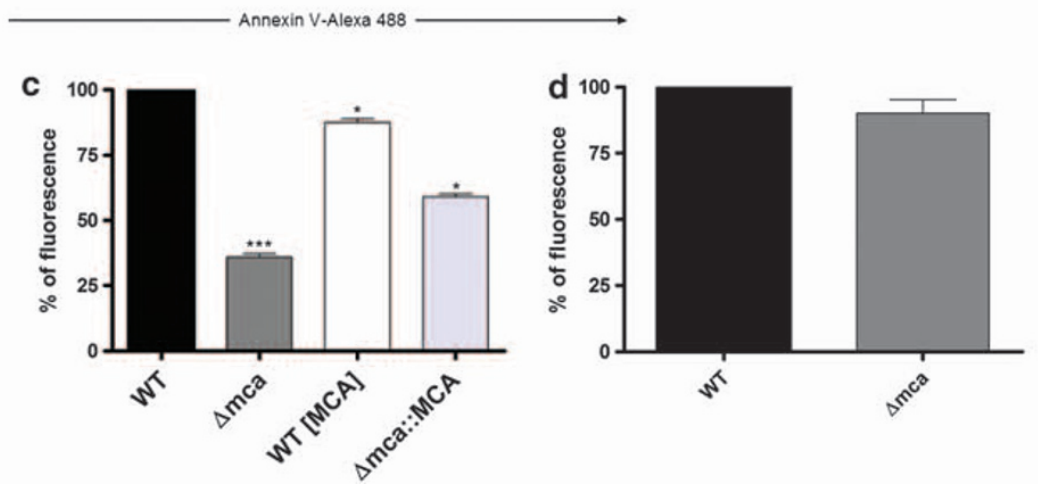

Figure 6 Drug-induced cell death and the mitochondrion. (a) Ann V labelling of MLF-treated parasites. Promastigotes were incubated with $20 \mu \mathrm{M} M L F$ for $15 \mathrm{~h}, \mathrm{co}$-stained with Annexin-V Alexa 488 and analysed by flow cytometry. Numbers represent the percentage of cells in each of the quadrants. Dot plots are representative of three independent assays. (b) Leishmanicidal activity of MLF and CPT. Cell viability of $L$. mexicana promastigotes incubated with increasing concentrations of drugs was determined using a 3-(4,5-dimethylthiazol-2-yl)-2,5-diphenyl tetrasodium bromide assay. Results are expressed as the drug concentration $(\mu \mathrm{M})$ necessary to inhibit parasite growth by $50 \%\left(\mathrm{IC}_{50}\right)$. Results are the mean $\pm \mathrm{s}$.d of six independent experiments. Significant differences compared with WT were determined by the Student's $t$-test $\left.{ }^{* \star *} P<0.001\right)$. Determination of mitochondrial membrane potential using TMRM. L. mexicana promastigotes (c) or axenic amastigotes (d) were incubated with TMRM and analysed by flow cytometry to measure mitochondrial membrane potential. Data are shown as the percentage of mitochondrial membrane potential compared with WT; a representative graph out of three independent experiments is shown. ${ }^{* \star *}$ and ${ }^{*}$ significant differences compared with WT (t-test, $\left.{ }^{* \star \star} P<0.001,{ }^{\star} P<0.05\right)$

an intact mitochondrion as assessed by tetramethylrhodamine methyl ester perchlorate (TMRM) labelling (data not shown). When the mitochondrial membrane potential of $\Delta m c a$ axenic amastigotes was investigated, no significant differences compared with WT were found (Figure 6d).

Previous studies have reported a role for yeast MCA in clearance of insoluble aggregates, ${ }^{25}$ but we could not detect any alteration in autophagy in $\Delta m c a$ promastigotes (Supplementary Figure S2), suggesting that MCAs do not have a role in clearance of protein aggregates via autophagy or in the induction of autophagic cell death. TSN was identified as a common substrate for both Norway spruce MCA and human caspase- $3,{ }^{22}$ but our studies indicate that TSN is not a substrate for L. mexicana MCA (Supplementary Figure S3). It is well established that Leishmania releases virulence factors that promote parasite survival in macrophages, ${ }^{4,34}$ so we tested whether L. mexicana MCA is secreted and thereby modulates functions of the host cell to promote Leishmania replication. No release of MCAs was detected (Supplementary Figure S4).

\section{Discussion}

We demonstrate in this study that MCAs can be successfully deleted from L. mexicana promastigotes, generating viable null mutants. We attribute this achievement, in contrast to our previous report on genetic manipulation of L. major MCA, ${ }^{27}$ to the use of a relatively new transfection method, the Nucleofector, which provides high transformation efficiency while being protective of cellular function. Indeed, we have now used the same methods to generate $L$. major MCA null mutants (data not shown), showing that there is not a noteworthy difference in the requirement for MCAs in the two species. The absence of MCA in L. mexicana did not produce an observable procyclic promastigote cell growth phenotype, as cells were viable and grew at a rate comparable to WT. $\Delta$ mca parasites also differentiated to amastigotes, both axenically in vitro and in macrophages, showing that absence of MCA did not prevent life cycle progression.

The most striking phenotype noted for the $\Delta$ mca parasites was their replication as amastigotes. The proliferation of $\Delta$ mca amastigotes in macrophages was significantly higher than WT and this was also the case in mice (Figures 3 and 4). This growth regulation was not apparent in axenic amastigotes (Figure 1f), possibly a reflection of the growth rate in axenic culture (doubling time of $\sim 23 \mathrm{~h}$ ) being significantly higher than in macrophages in vitro (doubling time of $\sim 90 \mathrm{~h}$ ) or in mice. As a corollary, overexpression of MCAs in amastigotes resulted in a lower replication rate compared with WT, both in macrophages and in mice, and this 
phenotype was dependent on the proteolytic activity of MCAs. This suggests that MCAs act as an amastigote-specific growth suppressor, having a role in regulation of proliferation in the mammalian host. MCA in L. mexicana was shown to be located in small punctate structures in the cytoplasm of the parasite (Figure 2), which is a similar location to that determined for MCA in $L$. major. ${ }^{27,28}$ The $L$. major enzyme was also found in the mitochondrion ${ }^{27,28}$ and associated with the mitotic spindle, ${ }^{27}$ whereas the $L$. donovani MCA has been reported to reside in acidocalcisomes ${ }^{29}$ - a location we do not observe in L. mexicana. It is plausible that different processed forms of $\mathrm{MCAs}^{28}$ are targeted to distinct subcellular compartments and that the isoform with an intact $\mathrm{C}$-terminal domain is associated with the punctate structures. The finding that MCA is not released by either promastigotes or amastigotes (Supplementary Figure S4) correlates with this localisation data as well as a previous proteomic analysis of the Leishmania secretome that did not detect released MCAs, ${ }^{35}$ suggesting that MCAs either acts directly within Leishmania to influence amastigote growth or that it processes a secreted protein that influences macrophage function to promote the rate of parasite proliferation. Two Leishmania peptidases CBP and GP63 are known to be released into the macrophage, where they modulate host cell signalling and promote parasite survival, ${ }^{34,36-38}$ but these peptidases are thought to be activated by autocatalytic cleavage of an inhibitory prodomain during intracellular trafficking ${ }^{39,40}$ rather than as part of a proteolytic activation cascade. It is known that the MCA MCA3 of $T$. brucei processes MCA4, a catalytically inactive pseudopeptidase that acts as a virulence factor through modulation of parasite growth in the mammalian host. ${ }^{41}$ However, MCA pseudopeptidases are apparently absent from the $L$. mexicana genome ${ }^{42}$ and so different growth regulation mechanisms must exist in these trypanosomatids.

We proposed previously that stress-induced cell death of Leishmania might be mediated by the release of lysosomal enzymes after organelle disruption and that this could involve cleavage of MCAs by the released cathepsin-like cysteine peptidases. ${ }^{12}$ Support for this hypothesis arose from the finding that a significant level of MCA processing was observed after $\mathrm{H}_{2} \mathrm{O}_{2}$ treatment in $L$. major overexpressing MCAs. ${ }^{28}$ Such processing of $L$. major MCA influences its subcellular location, as the enzyme encodes a mitochondrial localisation domain and a cytosolic retention domain that together modulate the balance of MCAs between the mitochondrial and cytosolic compartments. ${ }^{28}$ In this study, we have also observed a significant level of processing of L. mexicana MCAs in the overexpressor cell line (Figure 1d), which exhibits reduced cell proliferation as axenic amastigotes, as intracellular amastigotes and in mice (Figures 1f, $3 c$ and $5 d$ ). The enzymatic activity of MCAs was crucial for the observed growth defects, as mutation of the two cysteine residues in the active site of MCAs, and which are crucial for enzymatic activity, ${ }^{19,27}$ resulted in cells with WT proliferation rates. A significant level of processing was also observed in the WT $\left(\mathrm{MCA}^{\mathrm{C201-202G}}\right.$ ) cell line (Figure 1d), which was not observed in a $\Delta m c a\left(\mathrm{MCA}^{\mathrm{C} 201-202 \mathrm{G}}\right.$ ) cell line (data not shown), suggesting that intermolecular cleavage by MCAs was occurring in WT $\left(\mathrm{MCA}^{\mathrm{C} 201-202 \mathrm{G}}\right)$. Overexpression of MCAs in WT parasites also enhanced sensitivity to $\mathrm{H}_{2} \mathrm{O}_{2}$ - induced cell death in both $L$. major ${ }^{28}$ and $L$. donovani, ${ }^{29}$ most likely through impairment of mitochondrial function. ${ }^{28}$ Our results with $L$. mexicana $\Delta m c a$, however, show that even though the MCA-deficient mutant has reduced mitochondrial membrane potential, as assessed with TMRM (Figure 6c), it is not more or less susceptible to cell death inducers such as $\mathrm{H}_{2} \mathrm{O}_{2}$ than WT L. mexicana. Thus, clearly Leishmania MCA is not essential for cell death processes even though cell death is exacerbated when MCA is overexpressed. ${ }^{28,29}$ This MCA effect could occur through disruption of the distribution of MCAs between the cytosol and mitochondrion due to uncontrolled cleavage of MCAs by the cathepsin-like cysteine peptidases released from the lysosome, or through inappropriate activation of the enzyme in either compartment, which results in cleavage of non-physiological substrates.

In conclusion, we provide evidence that MCA is not essential for Leishmania proliferation, and our data provide no support for the proposed role of MCAs in regulated cell death. Rather our results suggest that MCA acts as an amastigote-specific growth suppressor, thereby regulating proliferation of the parasite in the mammalian host. Such selfregulation of growth is likely to be crucial in the maintenance of the intensity of infection in the mammalian host and thus be contributing greatly to pathogenesis.

\section{Materials and Methods}

Parasites. L. mexicana (MNYC/BZ/62/M379) promastigotes were grown in HOMEM medium with $10 \%(\mathrm{v} / \mathrm{v})$ heat-inactivated foetal calf serum (FCS) at $25^{\circ} \mathrm{C}$ as described previously. ${ }^{43}$ In vitro culture of $L$. mexicana axenic amastigotes was performed in Schneider's Drosophila medium supplemented with 20\% (v/v) FCS, $\mathrm{pH} 5.0$, at $32^{\circ} \mathrm{C}$ in $5 \% \mathrm{CO}_{2}$ as described previously. ${ }^{44}$

Generation of $L$. mexicana transgenic cell lines. For targeted gene replacement of the L. mexicana MCA gene (LmxM.34.1580), the 452-bp DNA fragment comprising the $5^{\prime}$ flanking region plus $5^{\prime}$ end of $M C A$ was amplified by PCR from L. mexicana genomic DNA using the primer pair OL3099 (HindllI) and OL3092 (Sall; all oligonucleotides used in this study can be found in Supplementary Table S1). The $3^{\prime}$ flanking region of MCA was generated by PCR using the primer pair OL3020 (Xmal) and OL3021 (Bgnl). Using Hindlll/Sall and $X$ mal/Bgll digestion, the $5^{\prime}$ and $3^{\prime}$ flanks were sequentially ligated into pGL345, to yield the hygromycin-resistant construct pGL1922. The bleomycinresistant plasmid pGL1923 was generated from pGL1922, replacing the HYG cassette by Spel/BamHI digest. Clones of parasites resistant to hygromycin were generated after transfection with HindllI/Bgll-linearised pGL1922, and a second round of transfection with linearised PGL1923 generated hygromycing-bleomycin double-resistant clones. The absence of MCA was assessed by both PCR and Southern blot.

For the re-integration construct, MCA was amplified with OL3420 and OL3421 primers, subcloned in PGEM-T easy vector and, following digestion with Xhol/ BamHI, ligated in Xhol/Bgll-digested pGL631 (pRIB) ${ }^{45}$ to generate the re-expressor construct pGL2004. The integration cassette was obtained by digestion with Pacl/ Pmel before transfection, and clones were selected for puromycin resistance.

For episomal expression, MCA from $L$. mexicana was isolated from genomic DNA using OL3399 and OL3400 primers. The 1320-bp fragment was subcloned into Xbal/Xhol-digested pTEX vector to generate pGL1984. To obtain parasites overexpressing the active site mutant MCA (MCA ${ }^{\text {C201-202G }}$ ), the two cysteines in the catalytic site were mutated by site-directed mutagenesis using OL1841 and OL1842 primers, to produce the active site mutant construct pGL2022. Site-directed mutagenesis was performed using the QuickChange mutagenesis kit (Stratagene, La Jolla, CA, USA) according to the manufacturer's instruction, and confirmed by DNA sequencing.

Transfection procedure. Mid-log phase L. mexicana promastigotes were transfected with $10 \mu \mathrm{g}$ of plasmid or digested DNA by electroporation using the Nucleofector system with the Human T-Cell Nucleofector kit (Lonza, Basel, Switzerland). Briefly, $5 \times 10^{7} \log$-phase cells were harvested by centrifugation at 
$1000 \times g$ for $10 \mathrm{~min}$. The cell pellet was resuspended in $100 \mu \mathrm{l}$ of Human T Cell Nucleofector Solution, transferred to Amaxa electroporation cuvettes and mixed with $10 \mu \mathrm{g}$ of DNA. Cells were nucleoporated on program U-033 on the Nucleofector machine (Amaxa GmbH, Cologne, Germany). Following electroporation, cells were incubated in their culture medium overnight and transfectants were cloned by limiting dilution with the appropriate antibiotics: $50 \mu \mathrm{g} / \mathrm{ml}$ hygromycin B (Invitrogen, Paisley, UK), $50 \mu \mathrm{g} / \mathrm{ml} \mathrm{G418} \mathrm{(Calbiochem,} \mathrm{Nottingham,}$ UK), $10 \mu \mathrm{g} / \mathrm{ml}$ phleomybin (InvivoGen, San Diego, CA, USA), $50 \mu \mathrm{g} / \mathrm{ml}$ puromycin (Calbiochem).

Southern blot analysis. Genomic DNA of $L$. mexicana WT, heterozygotes and null mutants $(\Delta m c a)$ was purified using the de DNAeasy kit (Qiagen, Crawley, UK), digested with Agel/Sacll and transferred to Hybond-N membranes (GE Healthcare, Little Chalfont, UK) following standard procedures and as described previously. ${ }^{43}$ Blots were hybridised with an alkaline-phosphatase-labelled DNA probe (AlkPhos Direct labelling, GE Healthcare) comprising the $5^{\prime}$ flanking region used for targeted gene replacement, or $500 \mathrm{bp}$ of the MCA ORF.

Immunoblotting. For western blot analysis, $1.5 \times 10^{7}$ cells were loaded per lane of a 10\% NuPAGE Bis-Tris gel (Invitrogen) in MOPS running buffer and transferred onto Hybond-C nitrocellulose membranes (GE Healthcare). Primary antibodies against LmjMCA, ${ }^{27}$ HA (Roche, Mannheim, Germany), EF1- $\alpha$ (Millipore, Bedford, MA, USA) and BiP (gift from JD Bangs (University of Wisconsin, Madison, WI, USA)) were used at 1:100, 1:1000, 1:10000 and $1: 10000$, respectively. Horseradish peroxidase-conjugated anti-rabbit (Promega, Southampton, UK) was used at 1:5000, anti-rat (Millipore) and anti-mouse (Promega) were used at 1:10000. Chemiluminescence was detected using ECL plus (GE Healthcare) or Novex ECL (Invitrogen).

Immunoflurescence. Parasites were fixed in $1 \%$ formaldehyde, permeabilised with $0.1 \%$ Triton and neutralised with $0.1 \mathrm{M}$ glycine. Cells were attached to poly-lysine-treated slides and blocked with TB buffer (PBS with $0.1 \%$ Triton and $0.1 \%$ BSA). A 1: 100 dilution of $\alpha$-LmjMCA antibody in TB buffer was applied for $1 \mathrm{~h}$ at room temperature. After washing the slides three times with PBS, anti-rabbit Alexa Fluor 594-conjugated antibody (Molecular probes, Eugene, OR, USA) at $1: 2000$ in TB buffer was added and the slides were incubated for $1 \mathrm{~h}$ in the dark at room temperature. Cellular DNA was stained with $1 \mu \mathrm{g} / \mathrm{ml} \mathrm{DAPI} \mathrm{in} \mathrm{TB} \mathrm{buffer.} \mathrm{A}$ mounting solution (2.5\% 1,4-diazabicyclo(2.2.2)octane in 50\% glycerol) was then applied to the slides. Images were obtained using an Applied Precision DeltaVision Deconvolution microscope system (Applied Precision, Inc. Issaquah, WA, USA) fitted with a Photometrics CoolSnap HQ (Roper Scientific, Inc. Tucson, $A Z$, USA) camera. Fluorescence images were acquired with the RDTRPF filter $\left(\lambda_{\mathrm{Ex}} 555 \mathrm{~nm} / \lambda_{\mathrm{Em}} 617 \mathrm{~nm}\right)$ and DAPI filter $\left(\lambda_{\mathrm{Ex}} 360 \mathrm{~nm} / \lambda_{\mathrm{Em}} 457 \mathrm{~nm}\right)$. References images were obtained with the differential interference contrast filter.

Induction of cell death. Drug-induced cell death was attempted by incubating the parasites in the presence of $20 \mu \mathrm{M}$ MLF for $15 \mathrm{~h}$. For Ann V labelling $1.5 \times 10^{6}$ treated cells were washed with Ann V buffer (20 mM HEPES; $132 \mathrm{mM} \mathrm{NaCl} ; 3.5 \mathrm{mM} \mathrm{KCl} ; 0.5 \mathrm{mM} \mathrm{MgCl}$; pH 7.3, supplemented with $10 \mathrm{mM}$ glucose and $5 \mathrm{mM} \mathrm{CaCl}_{2}$ ) and incubated with Ann V-alexa 488 (Molecular Probes) for $15 \mathrm{~min}$ in the dark. Propidium iodine $0.4 \mu \mathrm{g} / \mathrm{ml}$ was added for $5 \mathrm{~min}$. Cold PBS was added to the samples, which were analysed in a BD FACScalibur (BD Biosciences, San Jose, CA, USA) equipped with an FL1 $(530 / 30 \mathrm{~nm})$ and FL2 $(585 / 42 \mathrm{~nm})$ filter. Drug sensitivities of promastigotes were determined using a 3-(4,5-dimethylthiazol-2-yl)-2,5-diphenyl tetrasodium bromide-based assay as described previously. ${ }^{46} \mathrm{IC}_{50}$ was the drug concentration required for half-maximal inhibition of the cellular growth rate.

Measurement of mitochondrial membrane potential. Log-phase promastigotes or axenic amastigotes were incubated with $50 \mathrm{nM}$ TMRM (Sigma, Gillingham, UK) for $30 \mathrm{~min}$ and analysed by flow cytometry. Fluorescence was detected in FL2. The experiment was performed in triplicate, normalised to $100 \%$ of WT.

Macrophage infectivity assay. PEMs were extracted from peritoneal lavage of female ICR mice. Cells were plated in Lab-teck 16-well chamber slides (VWR International Ltd, East Grinstead, West Sussex, UK) at a density of $5 \times 10^{4}$ cells/well and incubated overnight at $37^{\circ} \mathrm{C}$ and $5 \% \quad \mathrm{CO}_{2}$ in RPMI supplemented with $10 \%$ FCS. Cells were incubated with stationary-phase promastigotes at $5: 1$ promastigotes: macrophage ratio for $3 \mathrm{~h}$ at $32^{\circ} \mathrm{C}$ and $5 \%$ $\mathrm{CO}_{2}$, after which they were washed to remove extracellular parasites and were incubated for 24,72 or $120 \mathrm{~h}$ to allow intracellular replication. Cells were fixed for $20 \mathrm{~min}$ at $4{ }^{\circ} \mathrm{C}$ with $2 \%$ formaldehyde following permeabilisation with $0.1 \%$ Triton X-100 for 10 min and stained with Vectashield mounting media with DAPI (Vector Laboratories, Burlingame, CA, USA) to identify the parasites. The percentage of infected macrophages and number of amastigotes per infected macrophage were determined by examination of 200 macrophages per well, in quadruplicates, under fluorescence microscopy using a Zeiss Axioskop 2 (Zeiss Ltd, Cambridge, UK) fluorescence microscope.

Lesion-derived amastigotes were obtained from infected $B A L B / c$ mice, removing the footpad and rupturing it to homogeneity with the back of a syringe through a nylon membrane in RPMI supplemented with $10 \%$ FCS as described previously. ${ }^{44}$ Lesion-derived amastigotes or axenic amastigotes were used to infect macrophages at a $2: 1$ ratio or $1: 1$, respectively, for $3 \mathrm{~h}$. Extracellular parasites were removed by washing, and fresh medium was added. Cultures were maintained at $32^{\circ} \mathrm{C}$ and $5 \% \mathrm{CO}_{2}$ for 24,72 or $120 \mathrm{~h}$, after which cells were fixed and stained as stated above. For testing amastigote sensitivity to MLF, peritoneal macrophages were infected with stationary-phase promastigotes at a $5: 1$ ratio for $24 \mathrm{~h}$. Extracellular parasites were removed by washing and fresh medium containing $30 \mu \mathrm{M} \mathrm{MLF}$ was added. Cells were maintained at $32^{\circ} \mathrm{C}$ and $5 \% \mathrm{CO}_{2}$ for 24,72 or $120 \mathrm{~h}$, after which cells were fixed, stained and counted as stated above.

Infection of mice. Groups of six female BALB/c mice were inoculated in the right footpad with $2 \times 10^{6}$ stationary-phase $L$. mexicana promastigotes in PBS. Lesion development was monitored weekly over an 8-12-week period, until the footpad reached a thickness of $5 \mathrm{~mm}$, when mice were culled.

Statistical analysis. All data were analysed by the Student's $t$-test. The GraphPad Instat software (San Diego, CA, USA) was used to perform the analysis. Differences were considered significant when $P<0.05$. Significant differences are indicated as follows: ${ }^{* \star} P<0.001,{ }^{* *} P<0.01$ and ${ }^{*} P<0.05$.

Additional Materials and Methods can be found in Supplementary Information.

\section{Conflict of Interest}

The authors declare no conflict of interest.

Acknowledgements. We thank William Proto for his helpful comments on the manuscript. This work was supported by the Medical Research Council (grant number 0700127). The Wellcome Trust Centre for Molecular Parasitology is supported by core funding from the Wellcome Trust (085349).

1. Taylor RC, Cullen SP, Martin SJ. Apoptosis: controlled demolition at the cellular level. Nat Rev Mol Cell Biol 2008; 9: 231-241.

2. Debrabant A, Lee N, Bertholet S, Duncan R, Nakhasi HL. Programmed cell death in trypanosomatids and other unicellular organisms. Int J Parasitol 2003; 33: 257-267.

3. Murray HW, Berman JD, Davies CR, Saravia NG. Advances in leishmaniasis. Lancet 2005; 366: 1561-1577.

4. Kaye $P$, Scott $P$. Leishmaniasis: complexity at the host - pathogen interface. Nat Rev Micro 2011; 9: 604-615.

5. Lee N, Bertholet S, Debrabant A, Muller J, Duncan R, Nakhasi HL. Programmed cell death in the unicellular protozoan parasite Leishmania. Cell Death Differ 2002; 9: 53-64.

6. Das M, Mukherjee SB, Shaha C. Hydrogen peroxide induces apoptosis-like death in Leishmania donovani promastigotes. J Cell Sci 2001; 114: 2461-2469.

7. Holzmuller P, Sereno D, Cavaleyra M, Mangot I, Daulouede S, Vincendeau P et al. Nitric oxide-mediated proteasome-dependent oligonucleosomal DNA fragmentation in Leishmania amazonensis amastigotes. Infect Immun 2002; 70: 3727-3735.

8. Moreira MEC, Del Portillo HA, Milder RV, Balanco JMF, Barcinski MA. Heat shock induction of apoptosis in promastigotes of the unicellular organism Leishmania (Leishmania) amazonensis. J Cell Physiol 1996; 167: 305-313.

9. Paris C, Loiseau PM, Bories C, Breard J. Miltefosine induces apoptosis-like death in Leishmania donovani promastigotes. Antimicrob Agents Chemother 2004; 48: 852-859.

10. Sen N, Das BB, Ganguly A, Mukherjee T, Tripathi G, Bandyopadhyay S et al. Camptothecin induced mitochondrial dysfunction leading to programmed cell death in unicellular hemoflagellate Leishmania donovani. Cell Death Differ 2004; 11: 924-936.

11. Verma NK, Singh G, Dey CS. Miltefosine induces apoptosis in arsenite-resistant Leishmania donovani promastigotes through mitochondrial dysfunction. Exp Parasitol 2007; 116: 1-13. 
12. Zangger $\mathrm{H}$, Mottram JC, Fasel $\mathrm{N}$. Cell death in Leishmania induced by stress and differentiation: Programmed cell death or necrosis? Cell Death Differ 2002; 9: 1126-1139.

13. Reece SE, Pollitt LC, Colegrave N, Gardner A. The meaning of death: Evolution and ecology of apoptosis in protozoan parasites. PLoS Pathog 2011; 7: e1002320.

14. Ivens AC, Peacock CS, Worthey EA, Murphy L, Aggarwal G, Berriman M et al. The genome of the kinetoplastid parasite, Leishmania major. Science 2005; 309: 436-442.

15. Berriman M, Ghedin E, Hertz-Fowler C, Blandin G, Renauld H, Bartholomeu DC et al. The genome of the African trypanosome Trypanosoma brucei. Science 2005; 309: 416-422.

16. Uren GA, O'Rourke K, Aravind L, Pisabarro TM, Seshagiri S, Koonin VE et al. Identification of paracaspases and metacaspases: two ancient families of caspase-like proteins, one of which plays a key role in MALT lymphoma. Mol Cell 2000; 6: 961-967.

17. Vercammen D, van de Cotte B, De Jaeger G, Eeckhout D, Casteels $P$, Vandepoele K et al. Type II metacaspases Atmc4 and Atmc9 of Arabidopsis thaliana cleave substrates after arginine and lysine. J Biol Chem 2004; 279: 45329-45336.

18. Moss CX, Westrop GD, Juliano L, Coombs GH, Mottram JC. Metacaspase 2 of Trypanosoma brucei is a calcium-dependent cysteine peptidase active without processing. FEBS Lett 2007; 581: 5635-5639.

19. Gonzales IJ, Desponds C, Schaff C, Mottram JC, Fasel N. Leishmania major metacaspase can replace yeast metacaspase in programmed cell death and has arginine-specific cysteine peptidase activity. Int J Parasitol 2007; 37: 161-172.

20. Bozhkov PV, Suarez MF, Filonova LH, Daniel G, Zamyatnin AA Jr, Rodriguez-Nieto S et al. Cysteine protease mcll-Pa executes programmed cell death during plant embryogenesis. PNAS 2005; 102: 14463-14468.

21. McLuskey K, Rudolf J, Proto WR, Isaacs NW, Coombs GH, Moss CX et al. Crystal structure of a Trypanosoma brucei metacaspase. Proc Natl Acad Sci USA 2012; 109: 7469-7474.

22. Sundstrom JF, Vaculova A, Smertenko AP, Savenkov El, Golovko A, Minina E et al. Tudor staphylococcal nuclease is an evolutionarily conserved component of the programmed cell death degradome. Nat Cell Biol 2009; 11: 1347-1354

23. Tsiatsiani L, Van BF, Gallois P, Zavialov A, Lam E, Bozhkov PV. Metacaspases. Cell Death Differ 2011; 18: 1279-1288.

24. Lee RE, Puente LG, Kaern M, Megeney LA. A non-death role of the yeast metacaspase: Yca1p alters cell cycle dynamics. PLoS ONE 2008; 3: e2956.

25. Lee RE, Brunette S, Puente LG, Megeney LA. Metacaspase Yca1 is required for clearance of insoluble protein aggregates. Proc Natl Acad Sci USA 2010; 107: 13348-13353.

26. Coll NS, Vercammen D, Smidler A, Clover C, Van BF, Dangl JL et al. Arabidopsis type I metacaspases control cell death. Science 2010; 330: 1393-1397.

27. Ambit A, Fasel N, Coombs GH, Mottram JC. An essential role for the Leishmania major metacaspase in cell cycle progression. Cell death Diff 2008; 15: 113-122.

28. Zalila H, Gonzalez IJ, El-Fadili AK, Delgado MB, Desponds C, Schaff C et al. Processing of metacaspase into a cytoplasmic catalytic domain mediating cell death in Leishmania major. Mol Microbiol 2011; 79: 222-239.

29. Lee N, Gannavaram S, Selvapandiyan A, Debrabant A. Characterization of metacaspases with trypsin-like activity and their putative role in programmed cell death in the protozoan parasite Leishmania. Eukaryot Cell 2007; 6: 1745-1757.

30. Raina P, Kaur S. Knockdown of LdMC1 and Hsp70 by antisense oligonucleotides causes cell-cycle defects and programmed cell death in Leishmania donovani. Mol Cell Biochem 2012; 359: 135-149.

31. van Zandbergen G, Bollinger A, Wenzel A, Kamhawi S, Voll R, Klinger M et al. Leishmania disease development depends on the presence of apoptotic promastigotes in the virulen inoculum. PNAS 2006; 103: 13837-13842.
32. van Zandbergen G, Luder CG, Heussler V, Duszenko M. Programmed cell death in unicellular parasites: a prerequisite for sustained infection? Trends Parasitol 2010; 26: 477-483.

33. Williams RAM, Smith TK, Cull B, Mottram JC, Coombs GH. ATG5 is essential for ATG8dependent autophagy and mitochondrial homeostasis in Leishmania. PLoS Path 2012; 8: e1002695

34. Jaramillo M, Gomez M-A, Larsson O, Shio M-T, Topisirovic I, Contreras I et al. Leishmania repression of host translation through $\mathrm{mTOR}$ cleavage is required for parasite survival and infection. Cell Host Microbe 2011; 9: 331-341.

35. Silverman JM, Chan SK, Robinson DP, Dwyer DM, Nandan D, Foster LJ et al. Proteomic analysis of the secretome of Leishmania donovani. Genome Biol 2008; 9: R35.

36. Cameron P, McGachy A, Anderson M, Paul A, Coombs GH, Mottram JC et al. Inhibition of Lipopolysaccharide-induced macrophage IL-12 production by Leishmania mexicana amastigotes: the role of cysteine peptidases and the NF- $\mathrm{KB}$ signaling pathway. J Immunol 2004; 173: 3297-3304.

37. Halle M, Gomez MA, Stuible M, Shimizu H, McMaster WR, Olivier M et al. The Leishmania surface protease GP63 cleaves multiple intracellular proteins and actively participates in p38 mitogen-activated protein kinase inactivation. J Biol Chem 2009; 284: 6893-6908.

38. Contreras I, Gomez MA, Nguyen O, Shio MT, McMaster RW, Olivier M. Leishmaniainduced inactivation of the macrophage transcription factor AP-1 is mediated by the parasite metalloprotease GP63. PLoS Pathog 2010; 6: e1001148.

39. Brooks DR, Tetley L, Coombs GH, Mottram JC. Processing and trafficking of cysteine proteases in Leishmania mexicana. J Cell Sci 2000; 113: 4035-4041.

40. Ellis M, Sharma DK, Hilley JD, Coombs GH, Mottram JC. Processing and trafficking of Leishmania mexicana GP63: analysis using mutants deficient in glycosylphosphatidyl inositol protein anchoring. J Biol Chem 2002; 277: 27968-27974.

41. Proto WR, Castanys-Munoz E, Black A, Tetley L, Juliano L, Coombs GH et al. Trypanosoma brucei metacaspase 4 is a pseudopeptidase and a virulence factor. $J$ Biol Chem 2011; 286: 39914-39925.

42. Rogers MB, Hilley JD, Dickens NJ, Wilkes JM, Bates PA, Depledge DP et al. Chromosome and gene copy number variation allow major structural change between species and strains of Leishmania. Genome Res 2011; 21: 2129-2142.

43. Brooks DR, Denise H, Westrop GD, Coombs GH, Mottram JC. The stage-regulated expression of Leishmania mexicana CPB cysteine proteases is mediated by an intercistronic sequence element. J Biol Chem 2001; 276: 47081-47089.

44. Bates PA, Robertson CD, Tetley L, Coombs GH. Axenic cultivation and characterization of Leishmania mexicana amastigote-like forms. Parasitology 1992; 105: 193-202.

45. Mißlitz A, Mottram JC, Overath P, Aebischer T. Targeted integration into a rRNA locus results in uniform and high level expression of transgenes in Leishmania amastigotes. $\mathrm{Mol}$ Biochem Parasitol 2000; 107: 251-261.

46. Williams RAM, Tetley L, Mottram JC, Coombs GH. Cysteine peptidases CPA and CPB are vital for autophagy and differentiation in Leishmania mexicana. Mol Microbiol 2006; 61: 655-674.

Cell Death and Disease is an open-access journal published by Nature Publishing Group. This work is licensed under the Creative Commons Attribution-NonCommercial-No Derivative Works 3.0 Unported License. To view a copy of this license, visit http://creativecommons.org/licenses/by-nc-nd/3.0/

Supplementary information accompanies the paper on Cell Death and Disease website (http://www.nature.com/cddis) 